\title{
Characterizing Failure in Commercial Li-Ion Batteries with 4D X-Ray Microscopy
}

Jeff Gelb ${ }^{1,2}$, Paul Shearing ${ }^{3}$, Donal Finegan ${ }^{3}$, and Dan Brett ${ }^{3}$

1. San Jose State University, Department of Chemical and Materials Engineering, San Jose, CA, USA

2. Carl Zeiss X-Ray Microscopy, Inc., Pleasanton, CA, USA

${ }^{3 .}$ University College London, Department of Chemical Engineering, London, UK

Modern devices are increasingly reliant on portable energy storage solutions. With the growing popularity of hybrid and fully-electric vehicles, demands for high-capacity and long-life batteries are also increasing. Understanding how the batteries perform and how long they last is critical to making a successful electric vehicle, to ensure the safety and satisfaction of the consumer. In spite of this, failure modes of Li-ion batteries remain poorly understood. It is well-established that batteries decline in charge capacity with an increased number of charge cycles, but the precise nature of the degradation as well as the cause of their ultimate failure remains largely uncharacterized.

Here, we present the results of using a direct, non-destructive imaging method to reveal the structural component of failure modes of commercial Li-ion batteries [1]. Two commercially-sourced Panasonic NCR 18650B Li-ion battery cells were imaged using a Zeiss Xradia 520 Versa X-ray microscopy (XRM) system. The cells were initially surveyed with a $\sim 20 \mathrm{~mm}$ field of view, in order to show the 3D structure of the jelly roll and for bulk feature identification, as shown in Figure 1. One cell was then deconstructed in an inert environment and a small piece of each electrode was imaged with $\sim 200 \mathrm{~nm}$ resolution on a Zeiss Xradia 810 Ultra XRM. The higher resolution results, shown in Figure 2, revealed several features within the active layer, including cracks and voids within the particles. These cracks and voids represent a significant deviation from the idealized, structurally homogeneous models that are often incorporated into battery cell design, representing a unique finding about the real microstructure of the active particles. A second cell from the same supplier was then imaged with the same parameters as before, but several such volumes were virtually stitched together to show the internal geometry of the entire cell. From this result, a smaller region was imaged with $\sim 1.8 \mu \mathrm{m}$ resolution without opening the package, utilizing the non-destructive nature of X-ray imaging. The cell was then charge cycled 100 times at $0.5 \mathrm{C}$ and the capacity fade was recorded. This same cell was then re-imaged with the same parameters as before, both with the large field of view and high-resolution imaging modes. The resulting 3D slices showed the development of many cracks within the cathode layer, which are suspected to contribute to the ultimate failure of the battery cell.

Building on the results from previous studies, we aim to explore the changing microstructure of these complex materials and how this affects cell performance and lifetime [2]. This experiment represents the first observation in this effort, and future studies will further explore the relationship between microstructure, performance, and failure in greater detail.

\section{References:}

[1] A P Merkle and J Gelb, Microscopy Today (2013) pp. 10-15.

[2] D S Eastwood et. al., Advanced Energy Materials 4 (2013) p. 1-7. 


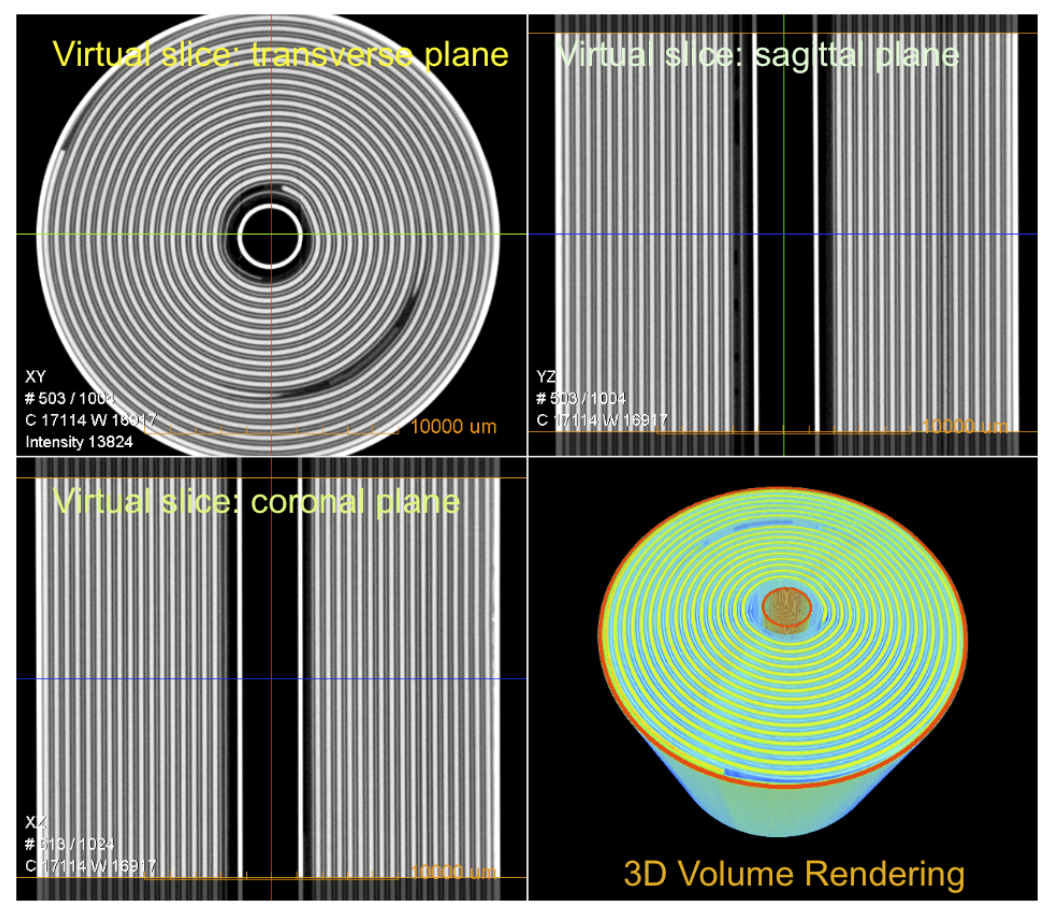

Figure 1. Multi-planar view of a commercial 18650 Li-ion battery, showing the different layers of jelly roll.

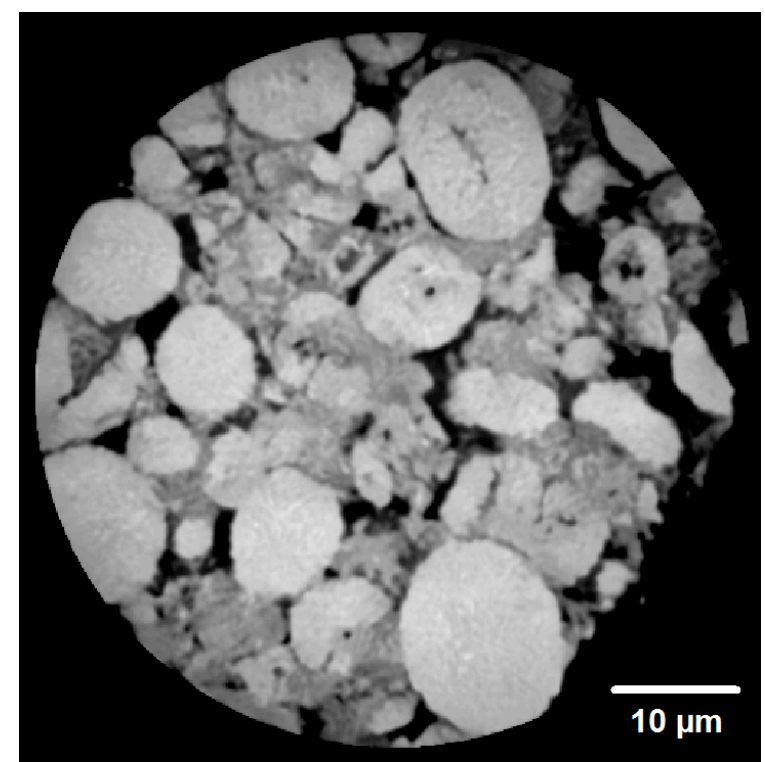

Figure 2. Virtual slice through a 3D volume of the cathode layer from a deconstructed commercial $18650 \mathrm{Li}$-ion battery as manufactured, imaged with $150 \mathrm{~nm}$ resolution. Several defects in the active particles are observed, including internal voids and cracks that extend into the pore network. 